\title{
Smart Safety Surveillance (3S): Multi-Country Experience of Implementing the 35 Concepts and Principles
}

\author{
Noha lessa ${ }^{1}\left[\right.$ - Viola Macolic Sarinic ${ }^{1}[1] \cdot$ Lilit Ghazaryan $^{2} \cdot$ Naira Romanova $^{2} \cdot$ Asnakech Alemu $^{3}$. \\ Watcharee Rungapiromnan ${ }^{4}$ (1) . Porntip Jiamsuchon ${ }^{4}$. Pattreya Pokhagul ${ }^{4}$. Jose Luis Castro ${ }^{5}$. \\ Diego Macias Saint-Gerons ${ }^{6}$ (D) $\cdot$ Gayane Ghukasyan $^{7} \cdot$ Mengistab Teferi $^{8} \cdot$ Madhur Gupta $^{9} \cdot$ Shanthi Narayan Pal $^{10}$ (1)
}

Accepted: 4 July 2021 / Published online: 31 July 2021

(c) The Author(s) 2021

\begin{abstract}
Introduction The Smart Safety Surveillance (3S) concept is based on the understanding that, when faced with competing pharmacovigilance priorities, countries will have to invest judiciously, by focusing on new priority products, sharing work and resources with other countries when possible and building national competence for those activities that cannot be delegated. Method The 3S principles were applied to Armenia, Brazil, Ethiopia, India, Peru and Thailand using three priority products: bedaquiline, rotavirus vaccine and tafenoquine. A baseline assessment of pharmacovigilance preparedness was used to identify gaps and establish a work plan. The impact was measured by comparing pre and post 3S-intervention outcomes, which included the number and quality of reports (completeness scores) in the WHO global database of Individual Case Safety Reports, VigiBase, and number of structural indicators met. The implementation period was 9-18 months, ranging from March 2018 (earliest started) until May 2020 (latest).

Result An increase in adverse drug reaction (ADR) reporting was demonstrated in Armenia (bedaquiline), Brazil (TB and malaria medicines), India (rotavirus vaccine) and Ethiopia (TB medicines). Completeness scores were above 0.5 at baseline in all countries, and reports improved in quality for Brazil (TB), Peru (malaria), Thailand (malaria) and India (immunization). The number of structural indicators met increased by more than double for Ethiopia. Ethiopia and India demonstrated an increased capacity for signal detection and signal evaluation. Armenia, Brazil, Peru and Thailand showed increased capacity to assess risk management plans following the implementation of $3 \mathrm{~S}$ principles.

Conclusion The $3 \mathrm{~S}$ concept has demonstrated success in different ways across the six countries. Activities focused on three products for a proof of concept of the $3 \mathrm{~S}$ principles, with the expectation that the project impact will be sustained through strengthened systems, to guide pharmacovigilance activities of other products in the future. It is important to continue monitoring the countries to understand if the gains and successes of the current $3 \mathrm{~S}$ project are sustainable.
\end{abstract}

\section{Introduction}

Access to medicines facilitated through expedited regulatory pathways and early access programmes has been supported by regulatory agencies such as the US Food and Drug Administration (FDA) and the European Medicines agency (EMA) from as early as 1992 [1, 2]. Often these programmes are based on limited clinical data to allow accelerated authorization into the market [3]. For example, the novel drug bedaquiline was granted a conditional license by the US FDA in 2010 for the treatment of multidrug-resistant tuberculosis before phase III clinical trials were complete [4]. Drug repositioning (also known as drug

Shanthi Narayan Pal

pals@who.int

Extended author information available on the last page of the article
Key Points

Investments in activities to strengthen countries' capacity to monitor the safety of novel medicines and vaccines can be optimized through the WHO Smart Safety Surveillance (3S) strategy, by focusing on priority products that are exclusive to the country and/or products with limited global experience.

The 3S concept was applied to six countries to enhance their ability to collect, assess and act on safety data on products of relevance to these countries. Although each country varied in the capacity to monitor safety of medicinal products at the start of the project, the study has demonstrated that the $3 \mathrm{~S}$ strategy can be applied equally to pharmacovigilance (PV) systems with different capacities. 
repurposing or drug reprofiling) is a process of developing new indications for existing drugs that have been streamlined for approval [5]. However, other safety issues may emerge after a drug is repurposed with changes in posology, when different patient groups such as the elderly and children may get the treatment; the adverse drug reaction (ADR) profile of the drug could change with more extensive use, when lesser known (or less frequent) adverse reactions may surface more prominently. Remdesivir, an antiviral originally designed for treatment of hepatitis $\mathrm{C}$, was repurposed for the treatment of Ebola in 2018 and repurposed again for the treatment of COVID-19 in 2020 [6-8]. Although such expedited processes for licenses in the EU and FDA are primarily for authorization in these countries and regions, low- and middle-income countries (LMICs) often leverage decisions from these reference agencies; the World Health Organization (WHO) also uses the decisions of such reference agencies to inform the work of its programmes such as the WHO prequalification programme, facilitating access to priority products in LMICs. Additionally, the EMA, in cooperation with the WHO, can provide scientific opinions on high priority human medicines, including vaccines, that are intended for markets outside of the European Union (EU). Expedited authorization (accelerated approval in the USA and conditional marketing authorization in Europe) for novel and often urgent treatments or vaccines might be given on the basis of several conditions, including timely completion of post-marketing studies, and should be able to rely upon robust and effective pharmacovigilance (PV) systems for a more thorough understanding and application of these products [9]. Monitoring requirements may be intensive and could even become a barrier for accessing new medicinal products in LMICs with very rudimentary or sub-optimal pharmacovigilance systems.

Given the finite resources at their disposal and the competing health priorities, LMICs would need to invest judiciously when it comes to pharmacovigilance. Rather than focus on general PV system development, the smarter option would be to focus their PV efforts on two types of medicinal products:

1. Products that are important to the country, introduced to address a high burden disease of public health priority and/or exclusive to the country (e.g. sleeping sickness).

2. Products with limited clinical data that will be introduced simultaneously, in high-income countries and in LMICs, with little global experience for LMICs to rely on.

These two scenario-based PV efforts can help prepare LMICs with a smart and agile PV system that is fit-forpurpose, fully ready to receive products that are new and/or exclusive to their own settings. In the absence of a focused, smart safety surveillance development plan, resources will continue to be used in a general way, without a meaningful and lasting return on investment. Above all, it is important that the absence of a functional PV system does not become a barrier to introducing new, life-saving medicinal products.

Additionally, global efforts to strengthen regulatory systems should include pharmacovigilance as an essential function within the regulatory framework [10]. For these reasons, many countries seek to invest in strengthening the PV system, to be recognized as a mature regulatory agency of international standards. However, with limited resources, it is essential to know which aspects of PV to invest in to gain maximum output/efficiency.

Smart Safety Surveillance (3S) is a pharmacovigilance risk prioritization strategy conceptualized by WHO to support Member States to identify which PV priorities to invest limited resources in, so that the system is prepared for the introduction of a new and/or an exclusive product. This paper will describe the challenges and achievements regarding the implementation of a $3 \mathrm{~S}$ approach in a selected group of countries. The objectives of this paper are to

- describe the application of the $3 \mathrm{~S}$ principles in a selection of countries using baseline assessments and country plans in preparation for the introduction of three priority medicinal products; and

- evaluate changes of selected variables and processes in the PV systems of targeted countries.

\section{Methodology: Application of 35 Principles}

Smart Safety Surveillance principles were applied in six countries about to introduce or having introduced three new products: bedaquiline (BDQ), tafenoquine (TFQ) and rotavirus vaccine (Rotavac ${ }^{\circledR}$, Bharat Biotech, India), for the treatment of multidrug-resistant $\mathrm{TB}$, malaria and prevention of rotavirus infections, respectively. A baseline assessment of PV preparedness was carried out in the six countries initially. Following this, national PV centres were supported in building their capacity and competence for identifying, assessing and adequately managing the risks associated with products in their markets (BDQ, rotavirus vaccine). For TFQ, a product yet to be introduced in the markets, national pharmacovigilance systems and competence were strengthened in anticipation of the product launch, using a proxy product with somewhat comparable profiles.

\subsection{Selection of Countries}

The six countries selected for testing the $3 \mathrm{~S}$ principles on $\mathrm{PV}$ systems were Armenia (BDQ), Brazil (BDQ and TFQ), Ethiopia (BDQ, TFQ), India (rotavirus vaccine), Peru (BDQ and TFQ) and Thailand (TFQ). Selection was based on burden 
of disease in LMICs and projected use of the new products. Advocacy at a national level was required and each of the selected six countries committed to implementation of $3 \mathrm{~S}$ interventions.

\subsection{Baseline Assessments}

WHO coordinated the development of an up-to-date global set of indicators using existing WHO, Indicator-Based Pharmacovigilance Assessment Tool (IPAT) and Global Benchmarking Tool (GBT) indicators, to assess PV preparedness of a country for the introduction of a new pharmaceutical product $[11,12]$. Baseline assessments of the PV infrastructure, competence, capacity and gaps in four of the target countries took place during scoping visits (prior to $3 \mathrm{~S}$ implementation): Armenia (March 2018), Ethiopia (February 2019), India (July 2018), Thailand (March 2019). For Brazil and Peru, the Global benchmarking tool and survey assessments conducted by Pan American Health Organization (PAHO), served as the baseline. Baseline assessments occurred prior to agreement to participate in the $3 \mathrm{~S}$ project. In all six countries the national pharmacovigilance centre is part of the national regulatory agency.

\subsection{Country Plans}

Using the baseline assessment, a holistic plan for PV was developed in each country according to the gaps identified in the baseline assessments to meet $3 \mathrm{~S}$ objectives. The work plans covered (i) infrastructure and framework for a functional PV system; (ii) qualitative and quantitative aspects of PV data including roles and systems for data management; (iii) data analysis, signal generation and validation (hypothesis testing); (iv) emphasis on the use of local PV data and analyses; (v) collaboration between PV centres and public health programmes. The project was implemented sequentially in the countries with a tailored approach over a period of 9-18 months during which selected outcomes were evaluated every 3 months from baseline assessments.

\subsection{S Implementation and Data Sources}

Technical support in implementing the $3 \mathrm{~S}$ workplans started in 2018, as a collaborative effort between WHO, and technical partners the Medicines and Healthcare products Regulatory Agency (MHRA, UK) and the Uppsala Monitoring Centre (UMC). The key support has been through training, workshops, training materials, study visits, direct support in implementing new IT technologies, tools and support for quality PV data management systems, infrastructure and capacity for data analysis and decision making. Information was obtained through VigiLyze, a tool used to access information from VigiBase. VigiBase is the WHO global database of Individual Case Safety Reports (ICSRs) maintained by the WHO Collaborating Centre for International Drug Monitoring, the UMC. To date (July 2020) there are over 26 million ICSRs originating from 148 Member States held in VigiBase. ICSRs come from a variety of sources and the probability that a suspected adverse effect is drug-related is not the same in all cases.

\subsection{Outcome Measures}

The following outcome measures were considered. Information on the outcome measures were extracted using VigiLyze and by querying the national PV centres. Outcomes were monitored until May 2020 at the latest.

- Changes from baseline in the number of structural indicators assessed using WHO PV preparedness tools before and after $3 \mathrm{~S}$ intervention.

- Changes from baseline in the reporting frequency for ICSRs at a national level of any drug aimed to treat malaria (Ethiopia, Thailand, Peru) or tuberculosis (Armenia, Brazil, Ethiopia), and the following specific medicines: bedaquiline (Armenia, Brazil and Ethiopia) and rotavirus vaccine (India). Since TFQ was yet to be introduced, primaquine was selected as a proxy because it is in the same drug class (8-aminoquinoline) and is indicated for the treatment of plasmodium vivax. Like TFQ, it can cause haemolytic anaemia in G6PD deficiency, which is one of the main risks of TFQ. The quality of ICSRs was evaluated using changes in VigiGrade ${ }^{\mathrm{TM}}$ completeness score. Completeness score is a quality measure comprising a number that accords a value to each field of clinically significant information contained in an ICSR. It starts at 1 for reports with information on time-to-onset, age, sex, indication, outcome, report type, dose, country, primary reporter and comments. It is reduced if information is missing and according to the clinical relevance of each missing dimension. An ICSR with a completeness score of $>0.8$ is considered to be well documented [13]. In the $3 \mathrm{~S}$ pilot, a completeness score of 0.5 was set as the minimum standard for quality of reports for selected countries, based on the average completeness score in the entire WHO global database of ICSRs (VigiBase). It should be noted that the minimum components of an ICSR are information on the reporter, country, suspected drug/vaccine and ADR. Having a high completeness score is the ideal needed to conduct a thorough clinical assessment, and ICSRs with a completeness score $<0.5$ are still valid. 
- Changes in activities related to signal detection, risk minimization plans and periodic safety update reports (PSURs).

\section{Results}

\subsection{Baseline Assessment and Application of 35 Principles}

Table 1 provides information on the number of structural indicators pre- and post-intervention assessed using the WHO PV preparedness tool. In total, there are 21 structural indicators. Armenia (16/21), India (20/21) and Thailand $(19 / 21)$ had most structures in place at baseline. Ethiopia met an additional 10 structural indicators following $3 \mathrm{~S}$ interventions. Structural indicators in Ethiopia include the drafting and submitting of the pharmacovigilance directive. Such a directive will ensure that there is a national policy or legislation for $\mathrm{PV}$ and that there are legal provisions for Marketing Authorization Holders (MAHs) to monitor and report the safety and quality of their products. This gives the PV centre authority so that processes can be actioned. Tables 1 and 2 list the activities that contributed to this.

As a result of activities listed in Tables 1, 2 and 3, over 500 people have been trained in $\mathrm{PV}$ in all six countries. The training focus varied between different countries due to differences in baseline PV capacity and gaps identified by the WHO PV preparedness tool. In Ethiopia and Peru, the focus was on reporting, analysis and signal detection while concepts around benefit/risk assessment, risk management plans (RMPs), PSURs and communication were the focus in Armenia, Brazil, India and Thailand.

Technological tools were enhanced in each of the six countries. Two countries introduced e-reporting and a mobile reporting app (Armenia and Ethiopia). Other countries modified existing data management systems so that they met international standards (E2B format in Brazil, Peru and Thailand); expanded access of the pharmacovigilance data management systems and databases to public health programmes (VigiLyze in Armenia and Ethiopia); formed reporting gateways/hubs to aid integration (Brazil), and used mobile technology to set up a coordination mechanism among national regulatory authorities (NRAs) and public health programmes using SMS (Brazil and Peru).

Brazil and Peru selected the cohort event monitoring methodology to monitor the safety of TB medicines. Countries were supported to develop specific guidelines, manuals and protocols, and to revise standard operating procedures that were out of date, missing or incomplete. These included a pharmacovigilance communication strategy, national PV guidelines and terms of reference for a Safety Review

Table 1 Structural indicators at baseline and after $3 \mathrm{~S}$ interventions for four countries following assessment using the WHO preparedness tool

\begin{tabular}{|c|c|c|c|}
\hline Country* & $\begin{array}{l}\text { Number of structural indica- } \\
\text { tors met prior to } 3 \mathrm{~S} \text { interven- } \\
\text { tion }\end{array}$ & $\begin{array}{l}\text { Number of structural indica- } \\
\text { tors met after } 3 \mathrm{~S} \text { interven- } \\
\text { tion }\end{array}$ & Example of activities conducted to meet structural indicators \\
\hline Armenia & $16 / 21(76 \%)$ & $17 / 21(82 \%)$ & $\begin{array}{l}\text { Reformation of Pharmacy Committee with PV experts } \\
\text { PV campaign and introduction of reporting technologies }\end{array}$ \\
\hline Ethiopia & $6 / 21(29 \%)$ & $16 / 21(76 \%)$ & $\begin{array}{l}\text { Revision of terms of reference for the Safety Review Committee } \\
\text { Draft and submission of PV directive } \\
\text { Regional centres, distribution of work so that there are enough staff } \\
\text { members to carry out PV functions } \\
\text { Making ADR reporting forms available (through technologies) in all } \\
\text { settings } \\
\text { Communication plan } \\
\text { Coordination procedures of PV activities with different stakeholders } \\
\text { Update of PV guidelines } \\
\text { SOPs for conducting PV activities } \\
\text { Existence of library or other reference sources for drug safety infor- } \\
\text { mation } \\
\text { PV courses organized by National PV Centre }\end{array}$ \\
\hline India & $20 / 21$ & $20 / 21$ & $\begin{array}{l}\text { Most structural indicators have been met, interventions focused on } \\
\text { public health and process indicators: integrating public health pro- } \\
\text { gramme and PV centre, and capacity for signal detection }\end{array}$ \\
\hline Thailand & $19 / 21$ & $19 / 21$ & $\begin{array}{l}\text { Most structural indicators have been met, interventions focused on } \\
\text { public health and process indicators: integrating malaria programme } \\
\text { with PV and building capacity to review PSURs and RMPs }\end{array}$ \\
\hline
\end{tabular}

$3 S$ Smart Safety Surveillance, $A D R$ adverse drug reaction, $P S U R s$ periodic safety update reports, $P V$ pharmacovigilance, $R M P s$ risk management plans, SOPs standard operating procedures, $W H O$ World Health Organization

*Baseline pharmacovigilance was assessed via the WHO Global Benchmarking tool in Brazil and Peru (see Table 2) 
committee (Armenia, Ethiopia); guidelines for good practice for vaccine safety (India); and a manual for data management systems for cohort event monitoring (Brazil).

In order to learn and gain new ideas from regulatory peers in the practice of signal detection, conducting expert safety committee meetings and assessing RMPs, staff from the national PV centres in Armenia, Ethiopia, India and Thailand were supported on study visits to other regulatory agencies (EMA, MHRA, TGA).

To strengthen collaborations between the public health programmes, other stakeholders such as the pharmaceutical industry and NGOs and the PV centre, stakeholders were invited to be part of the PV planning meetings and to join training workshops.

\subsection{Outcome Measures}

\subsubsection{Reports of ADRs with the use of priority products}

Table 4 and Fig. 1 show the number of ICSRs for BDQ, primaquine (chosen as a proxy for TFQ) and rotavirus vaccine in the selected periods before and after the $3 \mathrm{~S}$ implementation. Baseline assessment and implementation of $3 \mathrm{~S}$ principles occurred at different times between 2018 and 2019 in different countries. The information was obtained before and after 3S intervention. (Before 3S: baseline; after 3S: baseline +3 months, +6 months, +9 months, +12 months, +15 months, +18 months). 3S support commenced in March and July 2018 for Armenia and India, respectively, March and
February 2019 for Thailand and Ethiopia, respectively, and August 2019 for both Brazil and Peru.

As $3 \mathrm{~S}$ intervention commenced at different times in different countries, post-intervention data at 9 months are not yet available for Brazil and Peru, or at 12 months for Ethiopia and Thailand. Please note that agreement to participate in the $3 \mathrm{~S}$ initiative in Thailand occurred 3 months after baseline assessment.

In Armenia, there is a clear increase in the reporting frequency for BDQ at 18 months following $3 \mathrm{~S}$ implementation. This is also reflected in the total number of reports for TB medicines; hence, reporting has increased within the disease programme (Fig. 2). It is too early to know if a similar trend will be seen also in Brazil, Ethiopia or Peru for BDQ; however, there is a $50 \%$ increase in ICSRs for all TB medicines in Brazil 9 months after baseline (see Fig. 2).

Tafenoquine was not yet in the market at the time of the study, therefore it was not possible to obtain product-specific data. Instead, information on reporting for primaquine (PMQ) (as a surrogate for TFQ) is presented. There is a clear increase in reporting frequency for PMQ at 3 months following initiation of $3 \mathrm{~S}$ principles in Brazil. Reporting continues to rise and, at 9 months post-baseline, there is over a 200 -fold increase in the number of ICSRs for PMQ. This pattern is mirrored in reports for all antimalarials in Brazil. There is also an increase in the number of reports for antimalarials in Peru.

The number of ICSRs for rotavirus vaccine started to increase 6 months post-intervention. This increase is more marked amongst the number of ICSRs for all vaccines. The total number of ICSRs for all products has increased in India

Table 2 3S interventions for Brazil and Peru following assessment using the WHO Global Benchmarking tool

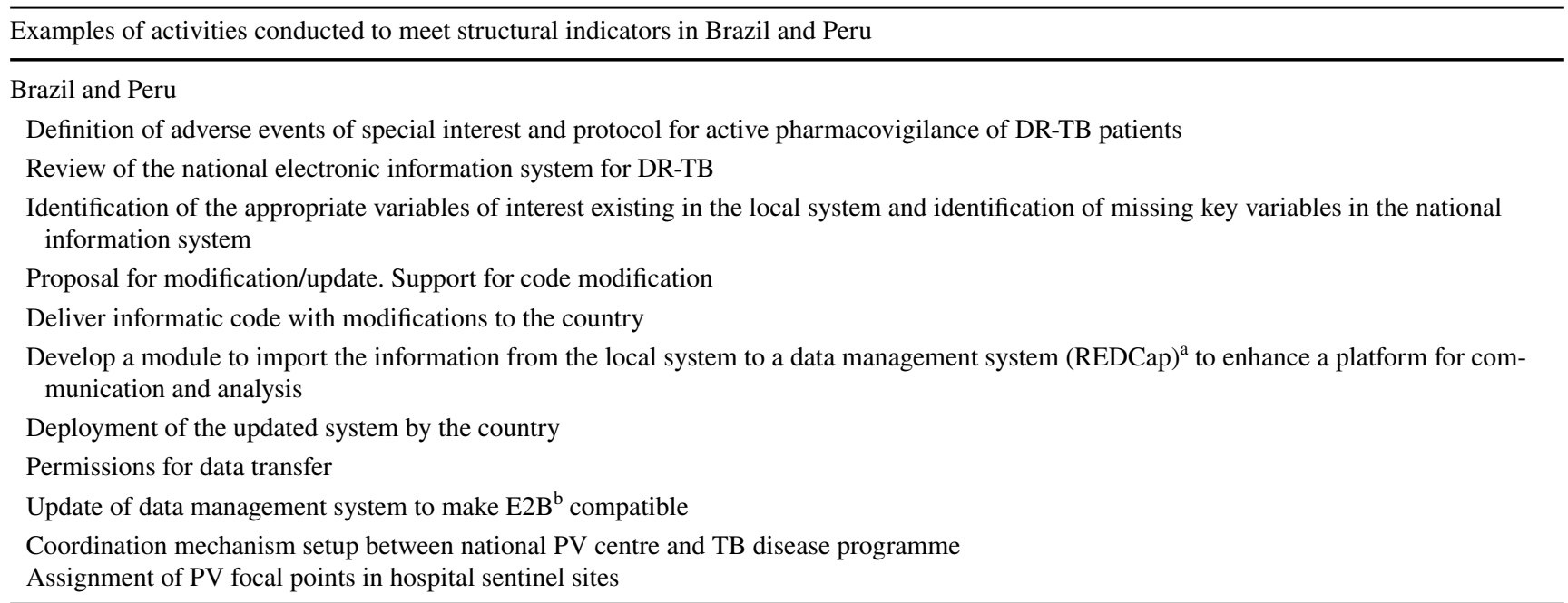

$3 S$ Smart Safety Surveillance, $D R-T B$ drug-resistant tuberculosis, $P V$ pharmacovigilance, $T B$ tuberculosis, $W H O$ World Health Organization ${ }^{a}$ REDCap (Research Electronic Data Capture) is a browser-based, metadata-driven EDC software and workflow methodology for designing clinical and translational research databases

${ }^{b}$ E2B: Data elements for transmission of individual case safety reports as per standards set by International Council for Harmonisation of Technical Requirements for Pharmaceuticals for Human Use (ICH) 
Table 3 Interventions made using the $3 \mathrm{~S}$ concept

\begin{tabular}{|c|c|}
\hline 3S Concept & Interventions made across the six countries \\
\hline Priority products & $\begin{array}{l}\text { Each country focused on one or two products according to disease burden and expected volume of use: } \\
\text { Armenia (BDQ), Brazil (BDQ and TFQ), Ethiopia (BDQ and TFQ), Peru (BDQ, TFQ), Thailand (TFQ) }\end{array}$ \\
\hline Holistic planning & $\begin{array}{l}\text { PV workplans for each country were devised } \\
\text { Strengthening activities at different points of the PV cycle included: } \\
\text { PV campaign and awareness workshops, events and media (Armenia, Ethiopia) } \\
\text { Training of signal detection and data analysis (all six countries) } \\
\text { Formation of regional centres or sentinel sites at hospitals, teaching materials, training the trainer on } \\
\text { PV and PV tools (Brazil, Ethiopia, Peru) } \\
\text { Training of healthcare professionals (all six countries) }\end{array}$ \\
\hline Tools and methods & $\begin{array}{l}\text { Reporting app launched (Armenia, Ethiopia) } \\
\text { Training on VigiFlow and VigiLyze (Armenia, Ethiopia, India) } \\
\text { VigiFlow provided in official country language (Armenia-Russia) } \\
\text { Active surveillance and cohort event monitoring (Brazil and Peru) }\end{array}$ \\
\hline Leveraging available resources & $\begin{array}{l}\text { Collaboration with NGOs, academics and public health programmes carrying out PV activities (All six } \\
\text { countries, e.g. Armenia and MSF) }\end{array}$ \\
\hline Reliance & $\begin{array}{l}\text { Regional protocol for active surveillance (Brazil and Peru) } \\
\text { Study visit to other regulatory agencies (Armenia, Ethiopia, India, Thailand) } \\
\text { Review of RMPs submitted by MAHs for appropriateness and feasibility (Brazil, Peru and Thailand) }\end{array}$ \\
\hline Work sharing & $\begin{array}{l}\text { Creation of platforms to share data between public health programmes and the PV centre, by expanding } \\
\text { access (restricted) to data management systems such as VigiFlow (Armenia, Ethiopia) } \\
\text { Upgrade of REDCap* data management system for data collection (Brazil and Peru) } \\
\text { Joint workshops (public health programme and PV centre) for signal detection and data analysis (Arme- } \\
\text { nia, Ethiopia, India, Thailand) }\end{array}$ \\
\hline
\end{tabular}

$3 S$ Smart Safety Surveillance, $B D Q$ bedaquiline, $M A H s$ Marketing Authorization Holders, $M S F$ Médecins Sans Frontières, NGOs non-governmental organizations, $P V$ pharmacovigilance, $R M P s$ risk management plans, $T F Q$ tafenoquine

*REDCap (Research Electronic Data Capture) is a browser-based, metadata-driven EDC software and workflow methodology for designing clinical and translational research databases

(6 months post-3S with respect to baseline), Thailand (at 9 months) and Brazil (steady increase post-3S) (Fig. 3).

\subsection{Reporting Quality}

Table 5 provides information on the average completeness score of ICSRs for malaria, TB and vaccines in the target countries.

At baseline, all countries had an average completeness score $>0.5$ for TB and malaria medicines, and rotavirus vaccine. Armenia and Ethiopia had an average completeness score $>0.8$ for TB medicines at baseline. However, the completeness score for TB medicines in Ethiopia dropped 12 months after baseline to 0.49 . Completeness scores increased 6 months post-baseline from 0.57 and 0.69 to 0.65 and 0.83 for Brazil and Peru, respectively. For malaria medicines, the average completeness score increased in Peru and Thailand from 0.62 and 0.55 to 0.92 and 0.75 , respectively, but decreased in Brazil (0.52-0.29).

\subsection{Signals and Risk Management Plans}

In India, PSURs for rotavirus vaccine have been analysed and it was concluded that the vaccine appears to be safe and well tolerated in healthy infants. Based on this information, the immunization programme in India concluded that the benefit of the vaccination to prevent the majority of rotavirus cases continues to outweigh its risks. A white paper providing information on the results of a self-controlled case series study (SCCS) investigating the risk of rotavirus vaccine and intussusception was published, and concluded that there was no increased risk of intussusception associated with rotavirus vaccine [17].

As tafenoquine was yet to be available, for capacity building purposes participants from Thai FDA reviewed the RMP for tafenoquine submitted by the MAH in other settings (e.g. Australia) after signing confidentiality agreements. The Thai FDA also reviewed safety information in the national pharmacovigilance database for signal detection and investigated three signals related to other antimalaria medicines. The signals were communicated to various stakeholders and 


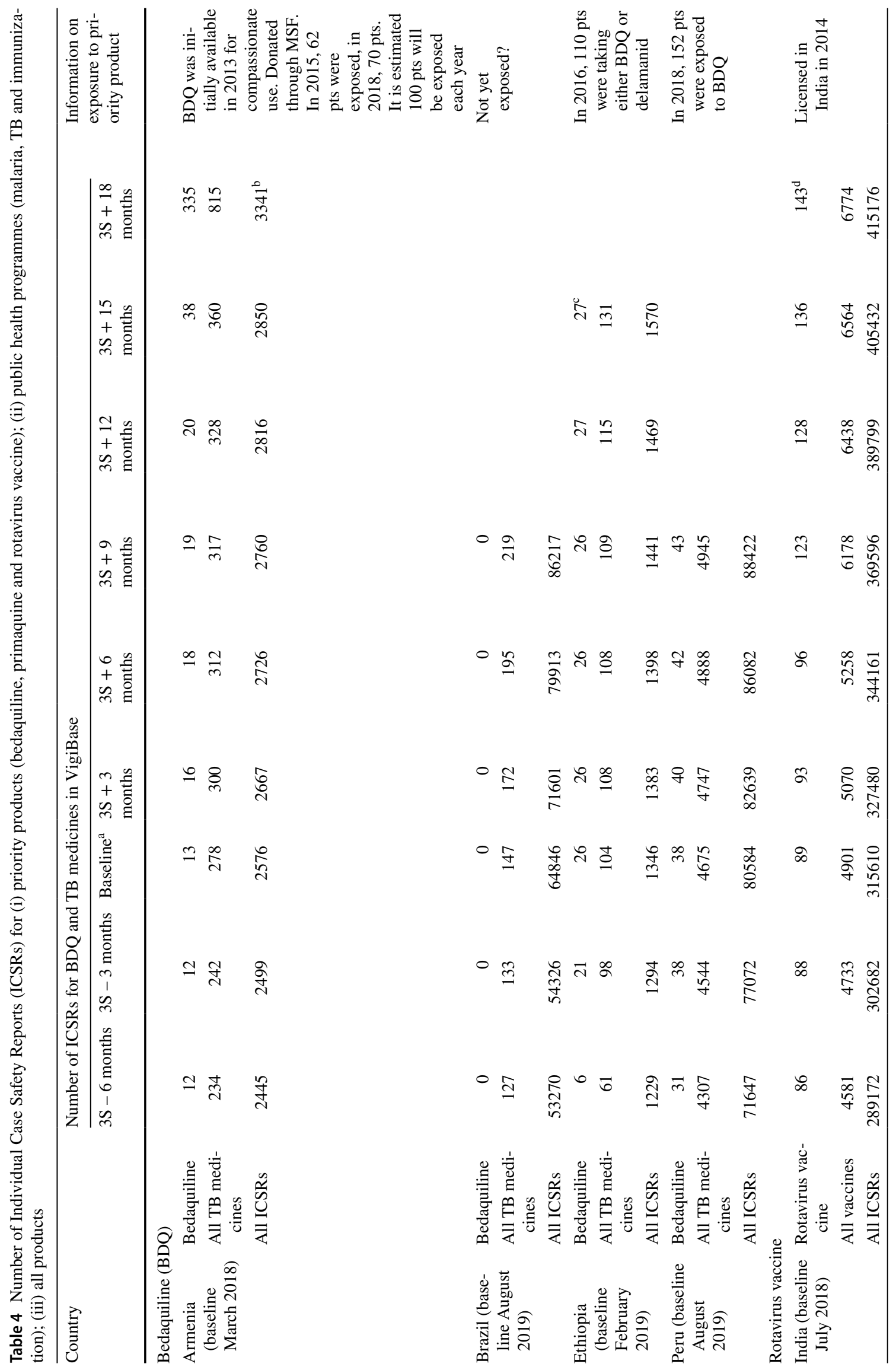




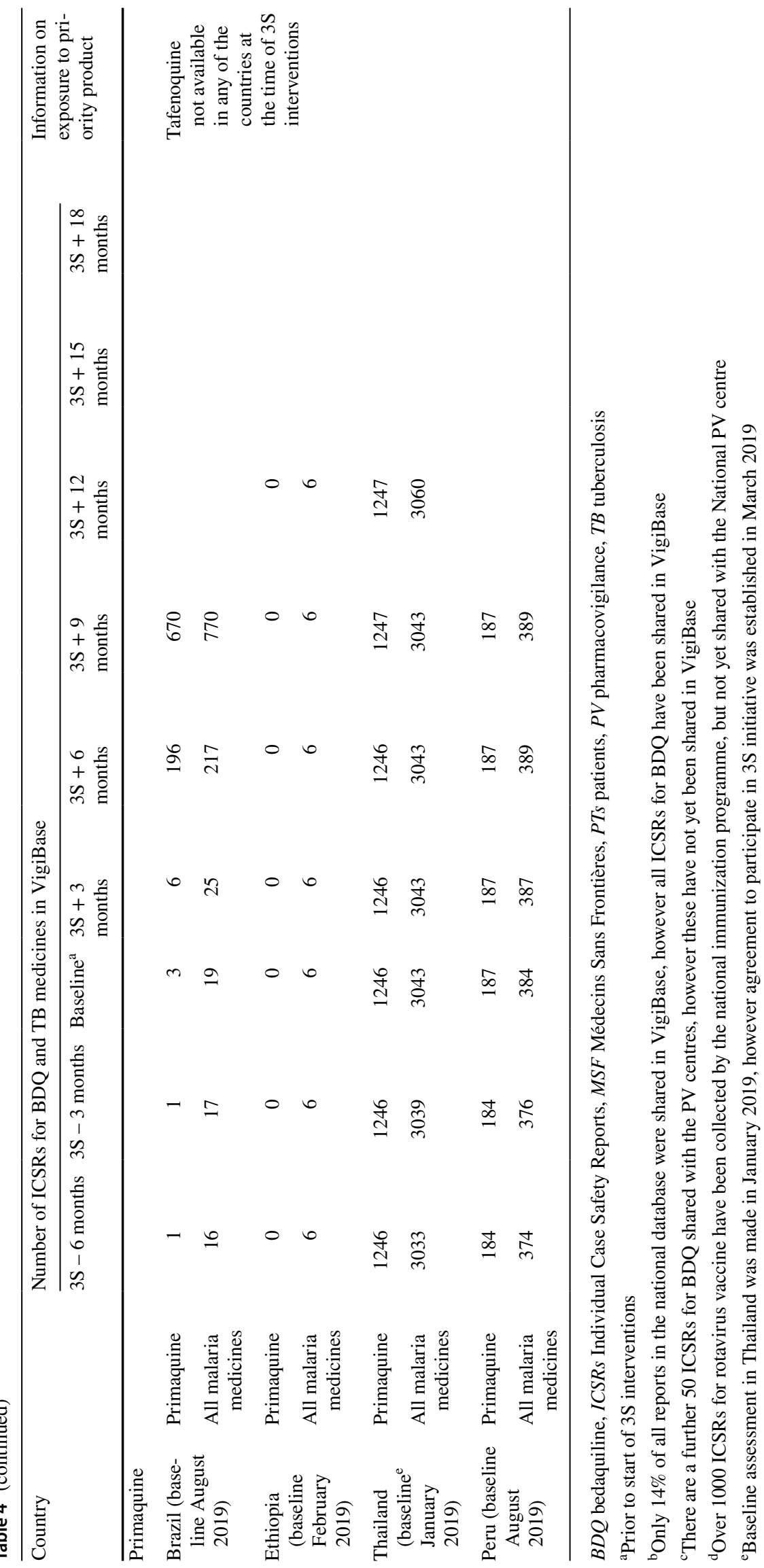


Fig. 1 Number of reports in VigiBase for the two priority products (BDQ, Rotavirus) and primaquine as a proxy for tafenoquine in target countries following $3 \mathrm{~S}$ intervention (up to 18 months post-baseline). $3 S$ Smart Safety Surveillance, $B D Q$ bedaquiline, ICSRs Individual Case Safety Reports, $P M Q$ primaquine

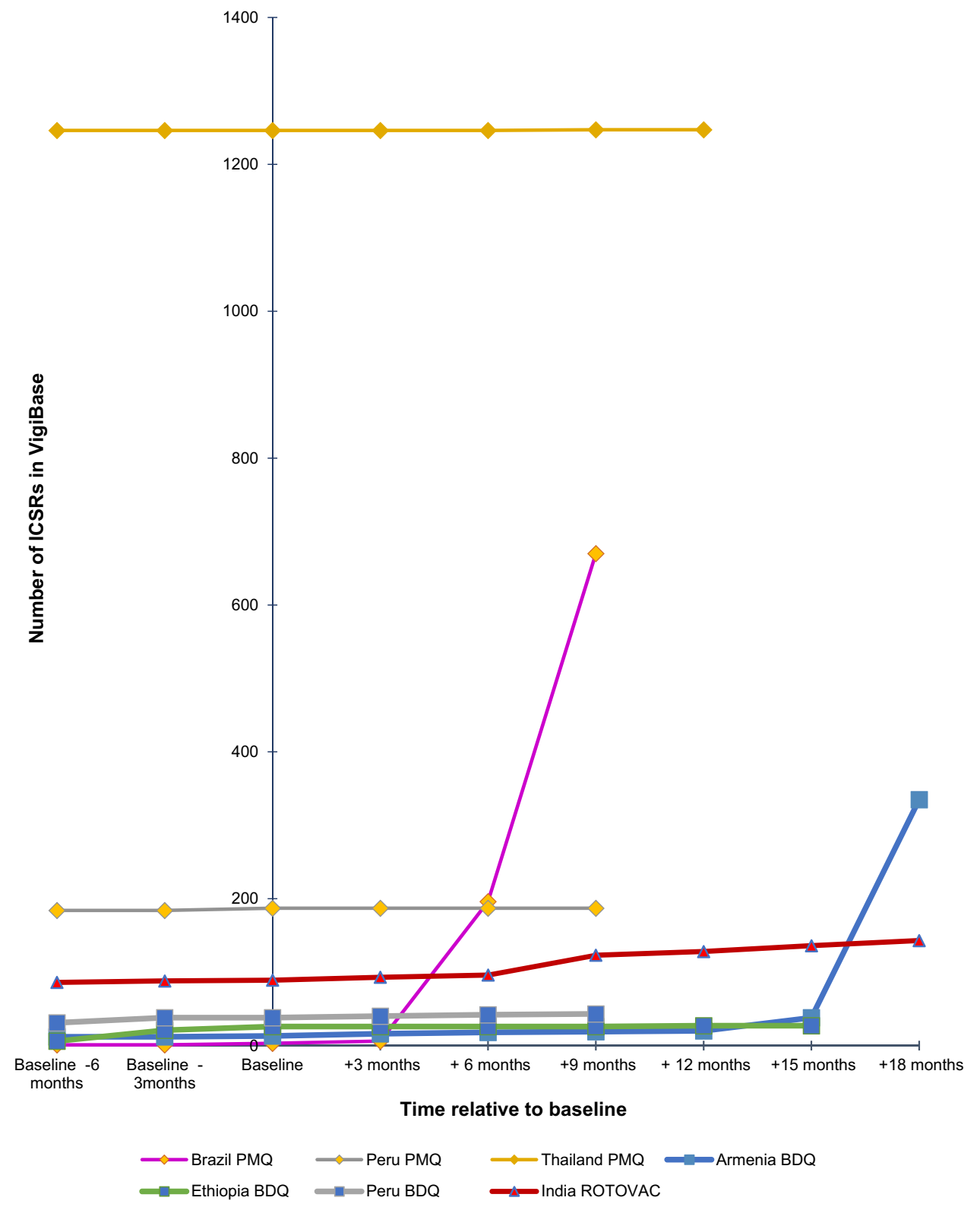

resulted in the revision of the Summary of Product Characteristics (SmPC) for primaquine and chloroquine.

In Armenia, the national PV centre is now performing PSUR assessments from $100 \%$ of the MAHs. The total number of signals detected pre- and post-3S workplan remained similar. The national PV centre is performing a descriptive analysis of the safety profile of BDQ.

In Ethiopia, qualitative causality assessments were made on all ICSRs for BDQ and both qualitative and quantitative case-by-case signal detection was carried out. Three potential signals were examined and were either not valid or evidence to support the signals were found to be weak.

\section{Discussion}

The 3S principles have been applied in different countries according to identified PV needs as assessed using the WHO PV preparedness tool. At baseline, Armenia, India and Thailand met a large proportion of structural indicators $(76 \%, 95 \%$ and $90 \%$, respectively). The focus of the $3 \mathrm{~S}$ strategy for these countries was on strengthening processes such as data analysis, benefit/risk assessments, evaluation of risk management plans and the integration with public health programmes to prevent parallel PV reporting systems. Ethiopia had fewer than half of the structural indicators in 
Fig. 2 Number of ICSRs in VigiBase for all antimalaria and TB medicines in the target countries following $3 \mathrm{~S}$ intervention (up to 18 months post-baseline). $3 S$ Smart Safety Surveillance, ICSRs Individual Case Safety Reports, $T B$ tuberculosis

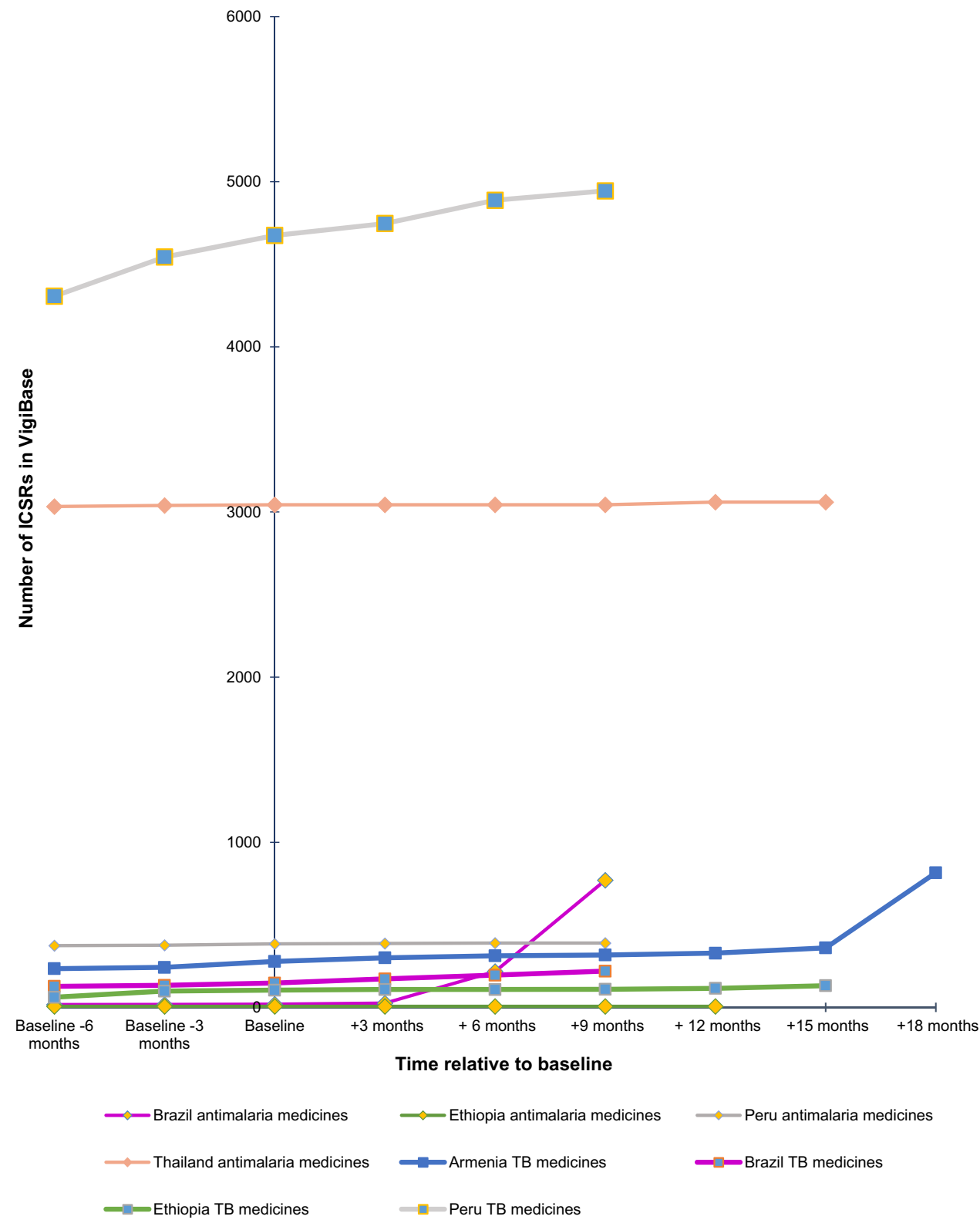

place at baseline, and the focus was on building these structures as well as strengthening existing processes. Using active surveillance methods, reporting tools and building a regional network were the approaches taken by Brazil and Peru [14-16]. The different approaches taken in these different countries reflect the differences in PV infrastructure and processes at baseline in the individual countries. An increase in outcome measures was expected, and included number of reports for priority products (BDQ, rotavirus vaccine) and/ or other medicines used in TB and malaria, total number of reports, reporting quality, signal detection and analysis of PSURs and RMPs. Implementation of $3 \mathrm{~S}$ helped to prioritize gaps, leading to a phased PV strengthening approach in line with WHO benchmarking assessments and institutional development plans in each of the different settings to attain longer term outcomes and regulatory maturity.

An increase in the number of ICSRs was observed in VigiBase for BDQ in Armenia, and with other TB medicines, PMQ and antimalarials in Brazil. A slight increase in ICSRs for rotavirus vaccine was observed in VigiBase for India 6 months post-3S initiation and the reporting quality improved as well.

In Armenia, increased collaborations with the national TB programme and other stakeholders such as Medicins Sans Frontieres (MSF) has led to data sharing of over 300 ICSRs for BDQ collected by MSF between 2015 and 2018. These ICSRs were later shared with the WHO global database of ICSRs, VigiBase, causing a peak in the number of ICSRs for BDQ 18 months post-3S initiation. There were 
Fig. 3 Number of all ICERs reported by target countries following $3 \mathrm{~S}$ intervention (up to 18 months post-baseline). $3 S$ Smart Safety Surveillance, ICSRS Individual Case Safety Reports

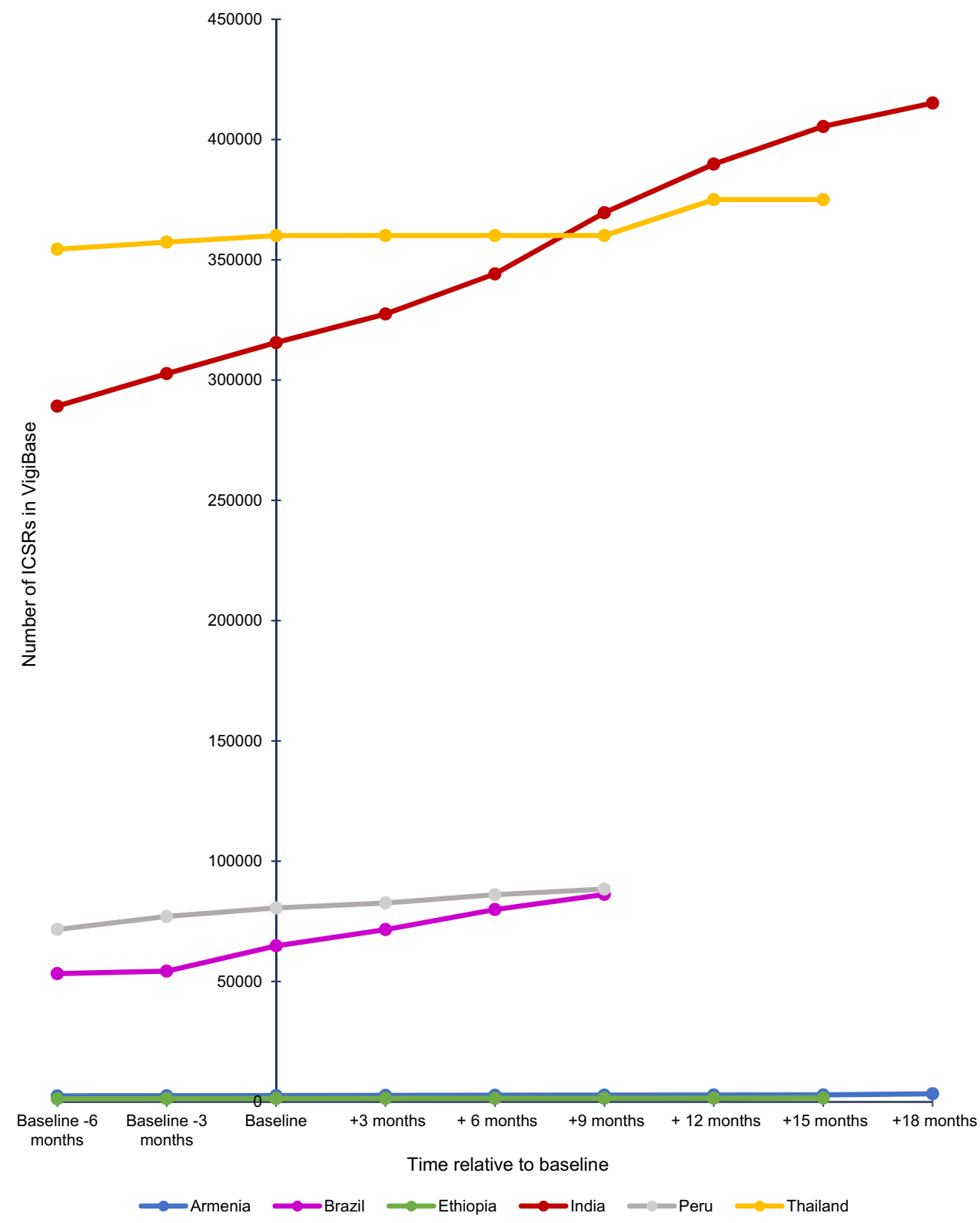

two main reasons for the 18-month lag-time for the ICSRs to be shared in VigiBase in Armenia: (i) a backlog of reports due to lack of data sharing between data collected by MSF through the national TB programme and the national PV centre prior to $3 \mathrm{~S}$ implementation and (ii) manual input of data into the data management system at the national PV centre. Other reasons that can cause delays are follow up of missing information and performing causality assessments before submission to VigiBase. The strengthened collaborations and new technologies introduced such as the mobile reporting app and electronic reporting are expected to reduce this lag time in the future. Monitoring of the impact of these technologies on the number of reports and time taken to share reports would be important, to evaluate the true impact of these tools. Additionally, if stakeholder engagement was facilitated at an earlier stage, the backlog of reports would have been prevented. For example, in the case of Armenia, MSF shared data 4 years after initiating active surveillance. Catching up with these reports was resource intensive.

The coordination mechanism between the National PV centre and the TB programme in Brazil and Peru consisted of using text messages for the follow up of ADRs as part of active surveillance methodologies (cohort event monitoring). Reports of adverse events were transferred to the national database for ICSRs. This resulted in an increase of ICSRs and quality of reports for TB medicines in Brazil. This approach was even more successful in increasing ICSRs for antimalarials, in particular, primaquine. However, the use of text messages for follow up resulted in a decrease in reporting quality in Brazil with ICSRs for primaquine, 
Table 5 Reporting quality at baseline, 6 and 12 months afterwards for $\mathrm{TB}$, malaria medicines and rotavirus vaccine in $3 \mathrm{~S}$ pilot countries

\begin{tabular}{|c|c|c|c|}
\hline Country & Baseline & $\begin{array}{l}6 \text { months after } \\
\text { baseline }\end{array}$ & $\begin{array}{l}12 \text { months } \\
\text { after baseline }\end{array}$ \\
\hline \multicolumn{4}{|c|}{ TB medicines } \\
\hline Armenia & 0.91 & 0.88 & 0.89 \\
\hline Brazil & 0.57 & 0.65 & \\
\hline Ethiopia & 0.8 & 0.73 & 0.49 \\
\hline Peru & 0.69 & 0.83 & \\
\hline \multicolumn{4}{|c|}{ Malaria medicines } \\
\hline Brazil & 0.52 & 0.29 & \\
\hline Ethiopia & 0.76 & & \\
\hline Peru & 0.62 & 0.92 & \\
\hline Thailand & 0.55 & & 0.75 \\
\hline \multicolumn{4}{|c|}{ Rotavirus vaccine } \\
\hline India & 0.69 & 0.6 & 0.72 \\
\hline
\end{tabular}

$3 S$ Smart Safety Surveillance, $T B$ tuberculosis

as there are limitations on how much information can be submitted via text messages.

The workshops on signal detection, and evaluation of PSURs and RMPs, besides enhancing capacity for these functions, were also significant in establishing the practice of joint activities: PV staff of the NRA and national disease specialists (malaria, TB, immunization programmes) worked together to complement each other's expertise in these joint activities. The pharmacovigilance centres in India and Thailand have started to be more confident when assessing PSURs and RMPs, and with signal detection and follow-up action. The joint reviews and advanced workshops were facilitated by technical partners from mature regulatory agencies. This and follow-on visits to other regulatory agencies (EMA, MHRA, TGA) facilitated peer-learning and the opportunity to observe good vigilance practices in different settings.

There was a slight increase in ICSRs for TB medicines at 15 months post-baseline (baseline: 104; 15 months post-3S: 131) from Ethiopia shared globally in VigiBase. Additionally, the PV centre had 50 ICSRs (at 15 months post-baseline) for BDQ that were provided by the national TB programme in Excel line-listing and had not yet been entered into the national data management system and VigiBase, causing a backlog. This could have been avoided if the use of PV reporting tools and integration of PV into the TB programme occurred earlier. Different channels to promote the use of these tools should also be explored. There is also a difference in the number of reports for TB medicines compared with malaria medicines, even though the same $3 \mathrm{~S}$ work plan was applied for both programmes. Product characteristics may very well have driven the number of reports of adverse events (with more events observed with BDQ, for example). However, it is also possible that in general there is less integration of $\mathrm{PV}$ in malaria than in the $\mathrm{TB}$ programmes in the country, contributing to the observed differences in reporting numbers. BDQ approval was subject to the condition that it would be monitored for safety, with a positive impact on number of ICSRs. The active Drug Safety Monitoring (aDSM) database was established by the WHO Global TB (GTB) Programme and the WHO Special Programme for Research in Tropical Disease (TDR). The database was to serve as a repository for safety data on new TB drugs and regimens. The quality and quantity of ICSRs for BDQ in the $3 \mathrm{~S}$ project may have been impacted by aDSM. While aDSM efforts had a positive knock-on effect on ICSR numbers for BDQ in the 3S project, the non-standardized Excel format used to transfer safety information from the national TB programme to the national PV centre could have contributed to the drop in completeness scores 12 months after baseline. BDQ has been supplied through donations with the support of NGOs that often have the obligation to conduct PV activities and report back to donors. In short, for BDQ a coordinated collaboration has existed between NGOs, national $\mathrm{PV}$, national TB programmes and $\mathrm{WHO}$, raising awareness for PV within the programme at the country level, albeit the parallel aDSM database at the global level. Similar collaboration and synergies between the various stakeholders should be considered for the integration of PV in malaria programmes in countries. In the other countries, in general, there was a mixed impact of $3 \mathrm{~S}$ on reporting quality. In some countries (India, Thailand and Peru) reporting quality increased, and in others it decreased (Brazil and Ethiopia). As stated earlier, the decrease in reporting quality could be due to loss of information through parallel reporting systems with non-standardized data formats, or incomplete data collection through the use of text messages. It should be noted that various overlapping PV strengthening projects such as the European and Developing Countries Clinical Trials Partnership (EDCTP)-funded PAVIA and PROFORMA initiatives are being implemented in Ethiopia and improvement in performance indicators can be attributed to the combined efforts of these three projects [18].

As tafenoquine has not yet been introduced in target countries, the full effects of $3 \mathrm{~S}$ intervention can only be assessed after the product becomes available and put into use. It is worth re-assessing outcome measures in the future, if and when tafenoquine becomes available for use, to evaluate the full impact of $3 \mathrm{~S}$ on PV of tafenoquine in Brazil, Peru and Thailand. The effects of $3 \mathrm{~S}$ on the impact of tafenoquine is expected to be demonstrated through the introduction of risk minimization measures, active surveillance activities and rapid signal detection of emerging safety concerns. Introduction of impact measures such as regulatory decisions and treatment policy using PV data related to the three products can take years to accomplish and was not feasible within the 
time course of this project, however, these measures would be important to monitor. On the other hand, changes to the PV structures and processes have been demonstrated, particularly in Ethiopia. It would be important to see whether these and other results from the $3 \mathrm{~S}$ project can be sustained in the long term, which will be a real measure of the success of the $3 \mathrm{~S}$ initiative.

\section{Conclusion}

The $3 \mathrm{~S}$ principles have been applied in six different countries with diverse PV capacity and varying requirements for preparedness. The diversity of the different countries limited the extent to which the results could be compared across the six countries. On the other hand, the pilot project has demonstrated that the $3 \mathrm{~S}$ strategy can indeed be applied to PV systems with different capacities. Various other initiatives with similar objectives of strengthening PV systems and capacity are also being implemented in parallel. The WHO Global Benchmarking approach that integrates PV as one of the key regulatory functions within an institutional development plan should provide the relevant framework to sustain the $3 \mathrm{~S}$ strategy. The results of these collective efforts should be reviewed in the near future.

Supplementary Information The online version contains supplementary material available at https://doi.org/10.1007/s40264-021-01100-z.

Acknowledgements WHO staff in country offices of Armenia, Brazil, Ethiopia, India, Peru and Thailand are acknowledged for their help in convening national pharmacovigilance centres and disease programmes, and for the operationalization of $3 \mathrm{~S}$ implementation. Implementation of $3 \mathrm{~S}$ was supported by collaborations with technical partners: the European Medicines Agency (EMA), the Medicines and Healthcare products Regulatory Agency (MHRA), UK; Therapeutic Goods Administration (TGA), Australia; and the Uppsala Monitoring Centre (UMC), Sweden. In addition, the following are acknowledged for their diligent work and support: Dr VG Somani, Drugs Controller General (India), CDSCO; Dr Eswara Reddy, Joint Drugs Controller (India), CDSCO; Ms Swati Srivastava, Deputy Drugs Controller, CDSCO, Ministry of Health \& Family Welfare, India; Dr MK Aggarwal, Deputy Commissioner (Immunization); Dr Deepak, Polpakra, Team Lead-Adverse Events Following Immunization, Ministry of Health \& Family Welfare, India, Dr V Kalaiselvan, Principal Scientific Officer, Indian Pharmacopoeia Commission; Dr Jai Prakash, Secretary cum Scientific Director (In-Charge), Ministry of Health \& Family Welfare, India in managing operations.

\section{Declarations}

Funding WHO support for implementation of the smart safety surveillance project through pharmacovigilance activities in countries was funded by the Bill and Melinda Gates Foundation.

Conflicts of interest/Competing interests Noha Iessa, Viola Macolic Sarinic, Lilit Ghazaryan, Naira Romanova, Asnakech Alemuu, Watch- aree Rungapiromnan, Porntip Jiamsuchon, Pattreya Pokhagul, Jose Luis Castro, Diego Macias Saint-Gerons, Gayane Ghukaysan, Mengistab, Teferi, Madhur Gupta and Shanthi Narayan Pal declare they have no conflict of interest.

Ethics approval Ethics approval was obtained from institutional ethical committees of all the participating countries for the active surveillance studies.

\section{Consent to participate N/A.}

\section{Consent for publication N/A.}

Availability of data and material (data transparency). The datasets generated during and/or analysed during the current study are available from the corresponding author on reasonable request.

Code availability VigiLyze ${ }^{\mathrm{TM}}$ : Information on number of reports and quality of reports from the WHO global database of Individual Case Safety Reports were obtained using VigiLyze.

Author contributions Conceptualization and design of work: SP. Data acquisition and analysis: SP, NI, VMS, LG, AA, WR, PJ, PP, JLC, GG, MF, MG. Interpretation of data: SP, NI, VMS, WR, JLC, DMSG. Drafting the manuscript: SP, NI. Revision for important intellectual content: SP, NI, VMS, LG, AA, WR, PJ, PP, JLC, DMSG, GG, MF, MG. All authors have read and approved the final version and agree to be accountable for all aspects of the work in ensuring that questions related to accuracy or integrity of any part of the work are appropriately investigated and resolved.

Open Access This article is licensed under a Creative Commons Attribution-NonCommercial 4.0 International License, which permits any non-commercial use, sharing, adaptation, distribution and reproduction in any medium or format, as long as you give appropriate credit to the original author(s) and the source, provide a link to the Creative Commons licence, and indicate if changes were made. The images or other third party material in this article are included in the article's Creative Commons licence, unless indicated otherwise in a credit line to the material. If material is not included in the article's Creative Commons licence and your intended use is not permitted by statutory regulation or exceeds the permitted use, you will need to obtain permission directly from the copyright holder. To view a copy of this licence, visit http://creativecommons.org/licenses/by-nc/4.0/.

\section{References}

1. US Department of Health and Human Services Food and Drug Administration. Guidance for industry expedited programs for serious conditions - drugs and biologics center for drug eEvaluation and Rrsearch. 2014. https://www.fda.gov/media/86377/downl oad. Accessed 23 Nov 2020.

2. European Medicines Agency. Accelerated assessment, https:// www.ema.europa.eu/en/human-regulatory/marketing-authorisat ion/accelerated-assessment. Accessed 23 Nov 2020.

3. Naci H, Salcher-Konrad M, Kesselheim A, Wieseler B, Rochaix L, Redberg RF, et al. Generating comparative evidence on new drugs and devices before approval. Lancet. 2020;395(10228):986-97.

4. Mahajan R. Bedaquiline: first FDA-approved tuberculosis drug in 40 years. Int J Appl Basic Med Res. 2013;3(1):1-2.

5. Parvathaneni V, Kulkarini NS, Muth A, Gupta V. Drug repurposing: a promising tool to accelerate the drug discovery process. Drug Discov Today. 2019;24(10):2076-85. 
6. Gilead. Development of Remdesivir. 2020. https://www.gilead. com/-/media/gilead-corporate/files/pdfs/covid-19/gilead_rdvdevelopment-fact-sheet-2020.pdf. Accessed 23 Nov 2020.

7. European Medicines Agency. First COVID-19 treatment recommended for EU authorisation. 2020. https://www.ema.europa. eu/en/documents/press-release/first-covid-19-treatment-recom mended-eu-authorisation_en.pdf. Accessed 23 Nov 2020.

8. US Food and Drug Administration. Coronavirus (COVID-19) Update: FDA issues emergency use authorization for potential COVID-19 treatment. 2020. https://www.fda.gov/news-events/ press-announcements/coronavirus-covid-19-update-fda-issuesemergency-use-authorization-potential-covid-19-treatment. Accessed 23 Nov 2020.

9. Cipriani A, Loannidis JPA, Rothwell PM, Glasziou P, Li T, Hernandez AF, et al. Generating comparative evidence on new drugs and devices after approval. Lancet. 2020;395(10228):998-1010.

10. Branigan D. WHO Benchmarking Tool made "more transparent" in evaluating regulatory authorities. Health Policy Watch. 2019. https://healthpolicy-watch.news/?s=WHO+benchmarking+tool. Accessed 23 Nov 2020.

11. Broojerdi AK, Sillo HB, Ostad RAD, Ward M, Refaat M, Parry J. The World Health Organization Global Benchmarking Tool aniInstrument to strengthen medical products regulation and promote universal health coverage. Front Med. 2020. https://doi.org/10. 3389/fmed.2020.00457.

12. Guzman J, O'Connell E, Kikule K, Hafner T. The WHO Global Benchmarking Tool: a game changer for strengthening national regulatory capacity. BMJ Glob Health. 2020;5(8):e003181.

13. Bergvall T, Noren GN, Lindquist M. VigiGrade: a tool to identify well-documented individual case reports and highlight systematic data quality issues. Drug Saf. 2014;37(1):65-77.

14. Pal SN, Duncombe C, Falzon S, Olsson S. WHO strategy for collecting safety data in public health programmes: complementing spontaneous reporting systems. Drug Saf. 2013;36(2):75-81.

15. Layton D, Shakir SA. Specialist cohort event Mmonitoring studies: a new study method for risk management in pharmacovigilance. Drug Saf. 2015;38(2):153-63.

16. Huang YL, Moon J, Segal JB. A comparison of active adverse event surveillance systems worldwide. Drug Saf. 2014;37(8):581-96.

17. White paper. Safety of rotavirus vaccine in India, smart safety surveillance approach. 2019. https://www.worldsdg2030.org/images/ White-paper-Book.pdf. Accessed 27 Apr 2021

18. Barry A, Olsson S, Minizi O, Bienvenu E, Makonnen E, Kamuhabwa A, et al. Comparative assessment of national pharmacovigilance systems in East Africa: Ethiopia, Kenya and Tanzania. Drug Saf. 2020;43:339-50.

\section{Authors and Affiliations}

\section{Noha lessa ${ }^{1}$ (1) Viola Macolic Sarinic ${ }^{1}$ (1) - Lilit Ghazaryan ${ }^{2} \cdot$ Naira Romanova $^{2} \cdot$ Asnakech Alemu $^{3}$. Watcharee Rungapiromnan ${ }^{4}$ (D) Porntip Jiamsuchon ${ }^{4} \cdot$ Pattreya Pokhagul $^{4}$. Jose Luis Castro ${ }^{5}$. Diego Macias Saint-Gerons ${ }^{6}$ (i) $\cdot$ Gayane Ghukasyan ${ }^{7} \cdot$ Mengistab Teferi $^{8} \cdot$ Madhur Gupta $^{9} \cdot$ Shanthi Narayan Pal ${ }^{10}$ (I)}

1 World Health Organization Headquarters, Geneva, Switzerland

2 Scientific Centre of Drug and Medical Technology Expertise of $\mathrm{MoH}$, Yerevan, Armenia

3 Ethiopian Food and Drug Administration, Addis Ababa, Ethiopia

4 Ministry of Public Health, Thai Food and Drug Administration, Nonthaburi, Thailand

5 Pan American Health Organization, Washington, DC, USA

6 INCLIVA-CIBERSAM, Valencia, Spain
7 WHO Country Office, Yerevan, Armenia

8 World Health Organization Country Office, Addis Ababa, Ethiopia

9 WHO Country Office, New Delhi, India

10 World Health Organization Headquarters, 20 Avenue Appia, 1202 Geneva, Switzerland 